\title{
Notas nomenclaturales sobre algunas gramíneas mediterráneas
}

\author{
Carlos Romero-Zarco', Juan Luis García-Castaño' \& Regina Berjano Pérez ${ }^{1,2}$ \\ 'Departamento de Biología Vegetal y Ecología, Universidad de Sevilla \\ ${ }^{2}$ Departamento de Botánica, Ecología y Fisiología Vegetal, Universidad de Córdoba
}

\author{
Correspondencia \\ C. Romero Zarco \\ e-mail: zarco@us.es \\ Recibido: 5 noviembre 2018 \\ Aceptado: 2 septiembre 2019 \\ Publicado on-line: 25 septiembre 2019 \\ Editado por: B. Cabezudo
}

\author{
Nomenclatural notes on some Mediterranean grasses \\ Palabras clave: Nomenclatura, tipificación, Gramineae, Poaceae, región \\ mediterránea
}

Key words: Nomenclature, typification, Gramineae, Poaceae, Mediterranean region
Durante la elaboración de los manuscritos destinados al volumen XIX de Flora iberica (Castroviejo, 1986 en adelante) se han resuelto algunas cuestiones de nomenclatura o tipificación que, siguiendo la tradición del proyecto, es preferible publicar previamente en formato de notas breves.

Airopsis tenella (Cav.) Asch. \& Graebn., Syn. Mitteleur. FI. 2(1): 298 (1899)

Millium tenellum Cav., Icon. 3: 37, t. 274 (1796) [basión.]

Airopsis globosa (Thore) Desv., Journ. Bot. (Desvaux) 1: 200 (1809)

Indicación locotípica: "Habitat in arenosis prope oppidum vulgo Pobla tornesa" [La Pobla Tornesa, Castellón].

Lectotipo: MA 475983!, designado aquí

Según el catálogo de los materiales de A. J. Cavanilles (cf. Garilleti, 1993) el ejemplar designado aquí como lectotipo es el único material original encontrado en el Herbario del Jardín Botánico de Madrid (MA). Paunero (1957: 189) citó material de esa localidad en su monografía sin indicar su número de registro en el herbario ni tipificación alguna. Hay otro material original, presumiblemente enviado por el mismo Cavanilles a Londres (LINN-Smith 121.9, "Spain-Cavanilles1793.-N. ${ }^{\circ} 8 . "$, cf. The Linnean Collections-Smith Herbarium, photographiam vidit) pero parece más recomendable elegir como lectotipo el material de la colección principal de Cavanilles, donde figura expresamente la localidad mencionada en el protólogo.

Arrhenatherum album subsp. fernandesii (Rivas Mart.) Romero Zarco, comb. et. stat. nov.
Basiónimo: Arrhenatherum fernandesii Rivas Mart. in Itinera Geobot. 15: 698 (2002)

Arrhenatherum erianthum var. montanum R. Fernandes in Mem. Soc. Brot. 6: 10 (1950) [syn. subst.]

Arrhenatherum album var. montanum (R. Fernandes) P. Silva in Agron. Lusit. 40: 6 (1980)

Indicación locotípica: "prope Caldeirão, circa Guarda" [actualmente embalse de Caldeirão en la Beira Alta]

Lectotipo: COI 9526 (photographiam vidit), designado aquí

Isolectotipi: LISI 3202-A!, LISU P 17030!, designados aquí

Las plantas de esta subespecie se caracterizan por la ausencia -o la reducción extrema- de los tubérculos basales que se desarrollan siempre en Arrhenatherum album subsp. album. Son frecuentes en las crestas cuarcíticas de la parte occidental del Sistema Central y en otras zonas occidentales de los valles del Duero, del Tajo y del Guadiana [Esp.: Ba Cc Sa Za. Port.: AAI BA TM]; al parecer se trata de un taxón diploide $(n=7$, cf. Devesa \& al., 1990 sub $A$. elatius subsp. sardoum, UNEX 8431!). Las características morfológicas de estas plantas y su distribución geográfica hacen más recomendable un tratamiento subespecífico dentro de A. album (Vahl) W.D. Clayton, en lugar del tratamiento específico o varietal de las anteriores propuestas. La existencia de un Arrhenatherum montanum (Dumort.) Potztal desaconseja el uso del epíteto montanum en este género. En su lugar se prefiere el epíteto fernandesii, anteriormente usado por Rivas Martínez en honor de la botánica que describió el taxón.

Arrhenatherum elatius subsp. cypricola $(\mathrm{H}$. 
Scholz) Romero Zarco, comb. nov.

Basiónimo: Arrhenatherum album subsp. cypricola H. Scholz in Willdenowia 37: 211 (2007)

Indicación locotípica: "Cyprus, Kaminaria, Platys valley, at the picnic site Komititzi, W of the summit Agios Ilias, dry open slope, 700 m."

Holotipo: Hand 4809, 5.5.2005 (B)

Taxón endémico de la isla de Chipre, que se caracteriza principalmente por tener el artejo de la raquilla muy corto (c. $0,4 \mathrm{~mm}$ ), carácter que lo aproxima más a $A$. elatius (L.) J. Presl \& C. Presl que a $A$. album. El lema de la flor superior es más densamente peloso que en otros taxones mediterráneos que se suelen incluir en esta especie, lo que tal vez hizo que $H$. Scholz se inclinara por clasificar esta subespecie en A. album, cuyas espiguillas sin embargo son muy diferentes debido al artejo de $(0,8) 1-2 \mathrm{~mm}$ que separa ambas flores (Romero-Zarco, 1985). Esta subespecie podría representar el taxón oriental de un conjunto de taxones mediterráneos vicariantes del que formarían parte $A$. elatius subsp. baeticum Romero Zarco en la cuenca mediterránea occidental y A. elatius subsp. nebrodense (Brullo, Miniss. \& Spamp.) Giardina \& Raimondo en Sicilia, todas ellas formas bulbosas diploides.

Arrhenatherum riofrioi Sennen, Diagn. Nouv.: 168 (1936)

Nombre aceptado: Arrhenatherum album (Vahl) W.D. Clayton var. album

Indicación locotípica: "Maroc: Melilla, Gurugu, ampelodesmatum, vers $1650 \mathrm{~m}$."

Lectotipo: Sennen \& Mauricio, F. Sennen PI. Esp. 1932, n 8557, BC 141849!, designado aquí

El exsiccatum de Sennen contiene plantas de pequeño porte y espiguillas relativamente pequeñas, idénticas a los diploides de la especie estudiados en los Sistemas Béticos (cf. RomeroZarco, 1985).

Avena filifolia var. glabra Boiss., Voy. Bot. Espagne 2: 655 (1844)

Nombre aceptado: Helictotricon filifolium (Lag.)

Henrard in Blumea 3: 430 (1940) var. filifolium

Avena filifolia Lag., Gen. Sp. PI.: 4 (1816)

Indicación locotípica: "varietas a in regione montanâ[...] Hab. species in Hispaniae regno Granatensi, Murcico (Lag.), in montibus Calabriae (Ten.), Siciliae (Guss.)."

Lectotipo: "Avena filifolia Lag. // dedit Lagasca"

(G-Boissier 165448!), designado aquí

Burdet \& al. (1981) no encontraron ningún material original correspondiente al protólogo. Boissier (I. c.) incluye dos sinónimos para la especie: "A. [Avena] fallax Ten. Guss. PI. rar. t. 9. A. convoluta
Presl”. El primer nombre corresponde a la especie que hoy llamamos Helictotrichon sempervirens (Vill.) Pilg. (cf. Index Synonymique de la Flore de France) y el segundo a $H$. convolutum (C. Presl) Henrard, ninguna de ellas presentes en España. A continuación separa dos variedades. La primera la denomina var. glabra, donde se podría interpretar que incluye los sinónimos citados para la especie, y la segunda la describe como var. velutina "in alpinâ Sierra Tejeda", actualmente aceptada como especie [Helictotrichon sarracenorum (Gand.) Holub]. Sin embargo, en las observaciones al pie de la descripción comenta que las plantas de Italia pertenecen probablemente a una tercera variedad. Por tanto, entre estos elementos del protólogo, solo las plantas observadas por él mismo en Andalucía y la referencia al tipo de Lagasca -"in Hispanieae regno Granatensi, Murcico (Lag.)"- deben tenerse en consideración para tipificar el nombre varietal. Se da la circunstancia de que el lectotipo designado por Röser (1989) para Avena filifolia Lag. es precisamente un ejemplar del herbario de Boissier que ya se comentó en la revisión del género para la Península Ibérica (Romero-Zarco, 1984): “Avena filifolia Lag. // dedit Lagasca". Parece lógico designar ese mismo ejemplar como lectotipo de la primera variedad de la especie, aunque eso suponga establecer su ilegitimidad.

Corynephorus articulatus (Desf.) P. Beauv., Agrost. 90 (1812)

Aira articulata Desf., Fl. Atlant. 1: 70 (1798) [basión.]

C. divaricatus auct. fl. europ., non Aira divaricata Pourr.

Indicación locotípica: "Habitat in arvis prope Mascar" [Muaskar, Argelia]

Lectotipo: P 00320332 (photographiam vidit), designado aquí

El género comprende seis especies de origen y distribución principalmente mediterránea, una perenne $-C$. canescens (L.) P. Beauv.- de más amplia distribución en Europa, y cinco anuales. Murbeck (1900) hizo un tratamiento -aún válidode las especies anuales del género, incluyendo las tres especies aceptadas presentes en la Península Ibérica [C. articulatus, C. macrantherus Boiss. \& Reut. y $C$. divaricatus (Pourr.) Breistr.], junto con otras dos especies del centro y del este de la región mediterránea, respectivamente: $C$. oranensis Murb. y C. deschampsioides Bornm. (sub C. laxus Murb.). Sin embargo, el trabajo de Murbeck no tuvo la repercusión que merecía, pues los autores posteriores consideraron una sola especie anual en el género: $C$. articulatus s. I. Este fue el caso de Breistroffer (1950), quien rescató Aira divaricata 
Pourr. de la sinonimia de Aira caryophyllea L. y propuso la combinación Corynephorus divaricatus (Pourr.) Breist. para sustituir a C. articulatus s.

I. De todos los epítetos específicos aplicables a las especies anuales de Corynephorus, el de Pourret resultó ser el más antiguo. Paunero (1956), haciéndose eco del trabajo de Breistroffer, reconoció dos especies anuales, aplicando el epíteto "divaricatus" de Pourret a lo que se había llamado anteriormente "articulatus" de Desfontaines. La tipificación de Aira divaricata fue realizada posteriormente por Laínz (1971: 3537), quien, al designar el lectotipo, alertó de que no era lo que venía llamándose "articulatus". Por su parte, Bor (1973) aceptó la tipificación de Laínz pero la malinterpretó, identificando Aira divaricata Pourr. con Aira articulata Desf. sensu stricto, y no sensu lato. De ahí que el binomio Corynephorus divaricatus haya sido usado impropiamente desde mediados del $\mathrm{s}$. XX por cierto número de autores. El tratamiento de Tutin (1980) siguió a Paunero y a Bor, y no a Laínz, agravando la confusión nomenclatural. La advertencia de Kerguélen (1983): "Corynephorus divaricatus // Ce nom semble correspondre à $C$. fasciculatus Boiss. \& Reut. et non à C. articulatus (Desf.) Beauv.", no impidió que el error se reprodujera en algunas floras (p. ej., Romero-Zarco, 1987).

Helictorichon pallens (Link) Couderc \& Guédès in Taxon 25: 188 (1976)

Avena pallens Link in J. Bot. (Schrader) 1799(2): 314 (1800) [basión.]

Indicación locotípica: "An der Serra da

Arrabida"

Lectotipo: Avena pallens. // N. Lusit. // Hoffmannsegg (HAL 107182, photographiam vidit) designado aquí

El lectotipo designado lleva, además de la etiqueta original, otra moderna donde figura el año 1795 como fecha de recolección. No obstante, la planta debió de ser recolectada en la primavera de 1798 , fecha en la que Hoffmannsegg y Link visitaron la Sierra de Arrábida, ya que en su primer viaje a Portugal, en 1795, el conde Hoffmannsegg llegó en otoño -la especie florece en primavera- y apenas si pudo visitar los alrededores de Lisboa por falta del salvoconducto adecuado, regresando a Alemania en la primavera de 1796 (Oliveira, 2014). La calidad de la imagen consultada (Virtual Herbaria JACQ) no ofrece dudas acerca de su identidad. En el herbario de C. Boutelou hay un ejemplar de Helictotrichon pallens (SEV-HHUS 10730, caja B006) correctamente identificado como Avena pallens Link "de Portugal" con letra de Claudio Boutelou (F.J. Salgueiro com. pers.). El pliego contiene una panícula fértil y una hoja basal. Tuvo que ser incorporado al herbario entre 1800 y 1830, y posiblemente fue adquirido en los jardines botánicos de Kew, París o Madrid (F.J. Salgueiro, com. pers.).

Helictotrichon thorei Röser in Schlechtendalia 19: 30 (2009)

Avena longifolia Thore, Prom. Golfe Gascogne: 92 (1810) [syn. subst.]

Indicación locotípica: no se señala expresamente, pero del texto se deduce que la planta se encontró en las landas entre Uza y Linxe, departamento de Landas, SO de Francia

Lectotipo: L 005032 (photographiam vidit), designado aquí

Röser publicó este nombre como "comb. nov.". Sin embargo, el basiónimo, Avena thorei Duby es ilegítimo por ser un nombre superfluo que incluye expresamente a Avena Iongifolia Thore; por tanto, según el artículo 6.14 del código de Shenzhen (Greuter \& Rankin Rodríguez, 2018), se debe interpretar como un nombre de reemplazo, siendo Avena longifolia Thore el sinónimo sustituido. Existe un Helictotrichon longifolium (Nees) Schweick. (publicado en 1937) que impide el uso del epíteto longifolia en el género. En las bases de datos consultadas se han localizado tres ejemplares que pueden formar parte del material original. En el Rijksherbarium de Leiden (Naturalis Diversity Center, Bioportal) hay dos ejemplares de la colección "Hb. Pers." [C.H. Persoon, contemporáneo de J. Thore] marcados con etiquetas rojas como posibles isotipos. En la etiqueta original (manuscrita) del pliego L 0050031 hay una diagnosis de una "Avena villosa N. (Thore)" diferenciándola de $A$. elatior y mencionando "sur las landes", sin mención expresa de Thore como recolector. El pliego L 0050032 lleva una etiqueta con letra diferente a la anterior en la que figura el nombre y lugar de publicación, el hábitat: "Sylvis inter...(ilegible)" y la frase "Misit Thore". En la base de datos del Natural History Museum de Londres hay un ejemplar (BM 001134032) procedente del herbario de J. J. Roemer (también contemporáneo de J. Thore), con etiqueta de letra casi idéntica a la del pliego L 0050032, en la que figura "Thore" como recolector y las palabras "Ericetis" y "julio". En la base de datos del Muséum national d'Histoire naturelle (París) no se ha encontrado material original (el más antiguo encontrado es de 1832). En ninguno de los ejemplares presuntamente originales aparece la fecha o la localidad del protólogo. Los pliegos BM 001134032 y L 005032 son los únicos que mencionan al autor de la especie como posible recolector, pero en el protólogo se indica "Maio, 
Junio, ericetis", lo que parece descartar al ejemplar de BM que es de julio. Designamos por tanto el pliego L 005032 como lectotipo.

\section{Trisetaria loeflingiana var. cavanillesii Paunero} in Anales Inst. Bot. Cavanilles 9: 529 (1951)

Trisetum cavanillesii Trin. in Mém. Acad. Imp. Sci. St.-Pétersbourg, Sér. 6, Sci. Math. 1: 63 (1831), nom. illeg. [syn. subst.]

Arundo pygmaea Spreng. in Mém. Acad. Imp.

Sci. Saint-Pétersbourg. Hist. Acad. 2: 299 (1810)

Indicación locotípica: [Spregel, op.cit:: 300]

"Habitat in monte Baldo prope Benacum lacum" [Alpes, $\mathrm{N}$ de Italia]

Paunero (1951) publicó su nombre varietal como combinación del nombre de Trinius. Sin embargo, el pretendido basiónimo es ilegítimo, ya que Trinius (1831) incluyó expresamente a Arundo pygmaea Spreng. en el protólogo. Por tanto, Paunero está creando en realidad un nuevo nombre en el rango varietal para sustituir al nombre de Trinius. Según el art. 58.1 del código de Shenzhen (Greuter \& Rankin Rodríguez, 2018) se puede tratar de un nombre de sustitución (con el mismo tipo que el nombre sustituido) o como un nuevo taxón con su propio tipo. En este caso, al igual que en el ejemplo Ex. 2 del citado artículo, se debe interpretar como nombre de sustitución en el rango varietal y llevaría por tipo el mismo del nombre ilegítimo al que sustituye, que a su vez lleva por tipo el mismo que Arundo pygmaea Spreng., pues Trinius no indica otro tipo distinto. En la publicación de su Trisetum cavanillesii, Trinius incluye dos sinónimos: "Avena Loefflingiana [sic] Cav. et Gaud." -se entiende que sensu Cavanilles y Gaudin, pues Cavanilles no crea ningún nombre nuevo y menciona a Linneo; no existe una "Avena loeflingiana Cav."-, y "Arundo pygmaea Sprgl. et Roemer \& Schultes", añadiendo como patrias "Helv. Ital.", en clara referencia a los sinónimos citados de Gaudin y de Sprengel, respectivamente. Arundo pygmaea es, según la lámina de Sprengel (1810: tab. VII), lo mismo que Paunero llamó Trisetaria loeflingiana var. cavanillesii, caracterizada por tener los pelos de la raquilla de mayor longitud hasta $2 \mathrm{~mm}$ en el ápice- y densidad que el tipo de la especie.

Trisetaria scabriuscula (Lag.) Paunero in Anales Inst. Bot. Cavanilles 9: 519 (1951)

Avena scabriuscula Lag. in Varied. Ci. 4(22): 212 (1805) [basión.]

Indicación locotípica: "Venit locis arenosis circa

Legionem urbem" [cf. M: Lagasca, Gen. Spec.

Plant.: 4 (1816)]

Lectotipo: designado de forma incompleta por
E. Paunero in Anales Inst. Bot. Cavanilles 9: 520 (1951): "León (Lagasca, typus, M.)"; lectotipo de segundo paso: "Avena scabrius- / cula // La Gasca. // varieds. // en Leon." (MA 8129!), designado aquí

Cebolla et al. (1997) indican "Typus: Lagasca (MA 8122), cf. Fernández Casas \& Gamarra (1993: 71 )" y reproducen el pliego en la página 65 . Sin embargo, lo que se lee en la etiqueta es "Reino de Valencia // $\mathrm{Herb}^{\circ}$ Cavanilles", localidad y recolector que están en serio conflicto con el protólogo. En realidad, Fernández Casas \& Gamarra (1993) se limitan a recoger el único pliego que encontraron en una selección de materiales presuntamente lagascanos bajo el nombre Avena scabriuscula y no tipifican nada: "En este trabajo no se lectotipifica en caso alguno" (op. cit.: 68). De acuerdo con el art. 9.17 del Código de Shenzhen (Greuter \& Rankin Rodríguez, 2018), la cita de E. Paunero (1951: 520): "León (Lagasca, typus, M.)" debe considerarse un primer paso de lectotipificación, ya que hay dos pliegos en el herbario del Jardín Botánico de Madrid (MA) con esos datos. En uno de ellos (MA 8127) una de las etiquetas originales lleva fecha de 1807, que resulta ser dos años posterior a la fecha de publicación del nombre, mientras que el otro (MA 8129), sin indicación de fecha, lleva, además de la etiqueta de Lagasca, una etiqueta más reciente manuscrita por Paunero con la indicacion "Specim. originalis". Se designa aquí como lectotipo de segundo paso el pliego MA 8129, que contiene alrededor de 36 ejemplares semejantes, con espiguillas glabras y aristadas. Un tercer pliego asociado a Lagasca (MA 8128), procedente de Villaobispo (León), podría también formar parte del material original.

Trisetum lasianthum Pérez-Lara in Anales Soc. Esp. Hist. Nat. 15: 401 (1886)

Nombre aceptado: Trisetaria dufourei (Boiss.) Paunero in Anales Inst. Bot. Cavanilles 9: 521 (1951)

Indicación locotípica: "in arenosis regionis calidae, in loco dicto Pinar de la Dehesilla prope Chiclana"

Lectotipo: "in arenosis loco dicto "Pinar de la Dehesilla' prope Chiclana, 10 Mai 1883" (MAF 29178!, etiqueta derecha, correspondiente al ejemplar en flor), designado aquí

La planta no difiere sustancialmente de la descrita por Boissier y no merece reconocimiento taxonómico. En la especie se observa cierta variabilidad en la densidad de la panícula que puede estar relacionada con el hábitat de la población, observándose panículas más laxas principalmente en hábitats cerrados, como pinares densos o matorrales altos. 
Trisetum viciosorum Sennen \& Mauricio in Sennen, Diagn. Nouv. 244 (1936)

Nombre aceptado: Avellinia festucoides (Link)

Valdés \& Scholz in Willdenowia 36(2): 662 (2006) Indicación locotípica: "Maroc: Atlas Rifain, meseta de Isaguen, $1600 \mathrm{~m}$." Lectotipo: BC 80866!, designado por RomeroZarco \& Sáez (2018: 168).

Se desestima este nombre para el tratamiento del género Trisetum en Flora Iberica.

\section{Agradecimientos}

Trabajo realizado en el marco del proyecto Flora iberica X(2) (CGL2015-66161-P) de la Dirección General de Investigación Científica y Técnica del gobierno de España (cofinanciado por FEDER) y parcialmente financiado por el Plan Andaluz de Investigación de la Junta de Andalucía (RNM 204). Agradecemos la colaboración prestada por los responsables y el personal técnico de los herbarios que han enviado el material estudiado, especialmente a los herbarios de la Universidad de Sevilla (SEV), Real Jardín Botánico de Madrid (MA) e Institut Botànic de Barcelona (BC).

\section{Bibliografía}

Bor, N.L. (1973). The typification of "Aira divaricata" Pourr. Webbia 27: 383-387.

Breistroffer, M. (1950). Notes sur deux plantes méditerranéennes. Procès-Verbaux de la Societé Dauphinoise d'Études Biologiques (Bio-Club) sér. 3 11: 3-4.

Burdet, H.M., A. Charpin \& F. Jacquemoud (1981). Types nomenclaturaux des taxa ibériques décrits par Boissier ou Reuter. 1. Gymnospermes à Graminées. Candollea 36: 543-584.

Castroviejo, S. (coord., 1986 en adelante). Flora iberica. Plantas vasculares de la Península Ibérica e Islas Baleares. Real Jardín Botánico, CSIC, Madrid.

Cebolla, C., J. A. López, J. M. Postigo \& M. A. Rivas (1997). Matritensis plantarum vascularium catalogus. Poaceae. Fontqueria 49: I-X, 1-85.

Devesa, J.A., T. Ruiz, R. Tormo, A. Muñoz, M.C. Viera, J.P. Carrasco, A. Ortega \& J. Pastor (1990). Contribución al conocimiento cariológico de las Poaceae en Extremadura (España) - II. Boletim da Sociedade Broteriana, série 2a , 63: 153-205.

Fernández Casas, J. \& R. Gamarra (1993). Herbarium lagascanum. Fontqueria 36: 67-108.

Garilleti, R (1993). Herbarium cavanillesianum. Fontqueria 38: [1-5] 6-248.

Greuter, W. \& R. Rankin Rodríguez (eds., 2018). Código Internacional de Nomenclatura para algas, hongos y plantas (Código de Shenzhen). Occasional papers from the Herbarium Greuter - 4, Berlín.

Kerguélen, M. (1983). Les Graminées de France au travers de "Flora Europaea" et de la "Flore" du C.N.R.S. Lejeunia, Nouv. Sér. 110: 79 pp.

Laínz, M. (1971). Aportaciones a la Flora Gallega VII. Madrid.
Murbeck, S.V. (1900). Contributions à la connaissance de la flore du nord-ouest de l'Afrique. IV. Graminaceae - Polypodiaceae. Lund (Suecia).

Oliveira, N. G. (2014). A Flore Portugaise e as viagens da Hoffmannsegg e Link a Portugal (1795-1801). Tesis doctoral, Universidad de Coimbra.

Paunero, E. (1951). Las especies españolas del género Trisetaria Forsk. Anales del Instituto Botánico A.J. Cavanilles 9: 503-582.

Paunero, E. (1956). Las Aveneas españolas I. Anales del Instituto Botánico A.J. Cavanilles 13: 149-229.

Paunero, E. (1957). Las Aveneas españolas II. Anales del Instituto Botánico A.J. Cavanilles 14: 187-281.

Romero-Zarco, C. (1984). Revisión del género Helictotrichon Besser ex Schultes \& Schultes fil. (Gramineae) en la Península Ibérica. I. Estudio taxonómico. Anales del Jardín Botánico de Madria 41(1): 97-124.

Romero-Zarco, C. (1985). Revisión del género Arrhenatherum Beauv. (Gramineae) en la Península Ibérica. Acta Botanica Malacitana 10: 123-154.

Romero-Zarco, C. (1987). Corynephorus Beauv. En B. Valdés, S. Talavera \& E.F. Galiano (eds.), Flora Vascular de Andalucía Occidental. Ketres Editores S.A., Barcelona.

Romero-Zarco, C. (1996). Contribución al conocimiento de las gramíneas del $\mathrm{N}$ de Marruecos. Lagascalia 18(2): 310-321.

Romero-Zarco, C. \& Sáez, L. (2018). Notas taxonómicas sobre el género Avellinia Parl. (Poacea: Poeae: Aveninae). Acta Botanica Malacitana 43: 167-169.

Sprengel, C. (1810). Graminum minus cognitarum. Decades duae. Mémoires de l'Académie impériale des sciences de St. Pétersbourg, 5e série, 2: 287-306.

Trinius, C. B. (1831). Graminum genera quaedam speciesque complures descriptinibus illustravit. Mémoires de l'Académie impériale des sciences de St. Pétersbourg, 6e série, Sci. Math. 1: 54-93 (1831).

Tutin, T.G. (1980). Corynephorus P. Beauv. En T.G. Tutin et al. (eds.), Flora Europaea 5: 231-232. Cambridge.

\section{Bases de datos en línea}

Index Synonymique de la Flore de France par M. Kerguélen. Institut National de la Reserche Agronomique. https://www2.dijon.inra.fr/flore-france/ index.htm [consultada en julio de 2019].

Muséum national d'Histoire naturelle. Vascular Plants (P). https://science.mnhn.fr [consultada en noviembre de 2018].

Natural History Museum, Botany collections [Londres]. http://www.nhm.ac.uk/our-science/collections/botanycollections.html [consultada en noviembre de 2018].

Naturalis Diversity Center, Bioportal. Nationaal Herbarium Nederland (actualizado periódicamente). https://science.naturalis.nl; http://bioportal.naturalis. $\mathrm{nl} /$ [consultada en noviembre de 2018].

The Linnean Collections. The Linnean Society of London. http://linnean-online.org [consultada en octubre de 2018]

Virtual Herbaria JACQ (2006 en adelante). Faculty Center, Botany and Botanical Garden, University Vienna. https://herbarium.univie.ac.at/database/index. php [consultado en octubre de 2018]. 